\title{
Trigonelline regulates Wnt/ $\beta$-catenin signaling in glomerular mesangial cells under high glucose conditions
}

\section{Chen Chen}

Jilin University

\section{Yan Shi}

Jilin University

\section{Zhen Chen}

Jilin University

\section{Xiangjun Li}

Jilin University

\section{Bo Sun}

Jilin University

Xiaoyan Yu

Jilin University

Jiulong Ma

Jilin University

Huayu Zhang

Jilin University

Xinran Ren

Jilin University

Ming Zhang

Jilin University

\section{Yan Zhao ( $790618540 @ q q . c o m$ )}

Jilin University Second Hospital

\section{Research}

Keywords: diabetic nephropathy, trigonelline, mesangial cells, Wnt/ $\beta$-catenin, apoptosis

Posted Date: April 20th, 2021

DOI: https://doi.org/10.21203/rs.3.rs-419018/v1 
License: (c) (i) This work is licensed under a Creative Commons Attribution 4.0 International License. Read Full License 


\section{Abstract}

Background: Trigonelline have hypoglycemic effects. In previous animal experiments, we observed that trigonelline (TRL) treat-ment attenuated metabolic abnormalities associated with hyperglycemic conditions in the experimental DN model. In streptozotocin (STZ)-induced rats, TRL treatment reduced albuminuria, lowered blood sugar, improved renal function and alleviated the pathological alterations within the glomerulus.

Methods: We stimulated human mesangial cells (HMC) with high glucose ( $30 \mathrm{mmol} / \mathrm{L})$ medium. HMCs were transfected with $\beta$-catenin plasmid or siRNA to investigate the effect of trigonelline on high glucoseinduced excessive proliferation and apoptosis of HMCs, and to understand its mechanism of action. Cell viability was measured by MTT assay. Flow cytometry was used to detect the cell cycle. Cell apoptosis was evaluated by flow cytometry and terminal dUTP transferase nick end labeling (TUNEL) assay. Protein and mRNA expression of $\beta$-catenin, Wnt5a, TCF4, Cyclin D1, and CDK4 were detected by western blotting and RT-PCR, respectively.

Results: Trigonelline inhibited cell proliferation by blocking cell-cycle progression at the $\mathrm{G} 1$ phase and decreased apoptosis via the Wnt/ $\beta$-catenin pathway. Elevated glucose levels enhanced the expression of $\beta$-catenin, an important modulator of diabetic nephropathy, while trigonelline restored up-regulation.

Conclusions: High glucose and high expression of $\beta$-catenin could lead to cell injury; however, this effect was mitigated by trigonelline via managing the canonical Wnt/ $\beta$-catenin signaling pathway.

\section{Introduction}

Diabetic nephropathy (DN) has become the third-largest threat to human health after cancer [1]. There is no specific treatment for $\mathrm{DN}$; the main therapy is a combination of hypoglycemic drugs and angiotensin receptor blockers or angiotensin-converting enzyme inhibitors. However, this therapy has disadvantages, including high costs, many side effects, and a long course of treatment [2-4]. Therefore, exploring the pathogenesis of DN and finding effective anti-DN drugs are of great importance. Pathological changes of DN include thickening of the basement membrane, accumulation of extracellular matrix, diffuse glomerular sclerosis, etc., the most serious development of renal failure [5-7]. Among them, the extracellular matrix is the main pathological change of mesangial cell proliferation. The accumulation of extracellular matrix and hypertrophy of mesangial cells eventually leads to glomerular sclerosis. [8]. Therefore, the function of mesangial cells plays an essential part in the clinical diagnosis and treatment of DN. Somebody has confirmed that chronic hyperglycemia promotes the molecular pathway leading to apoptosis and causes glomerular damage [9-11]. Therefore, hyperglycemia-induced excessive proliferation and apoptosis appear to be crucial factors in the progression of glomerulosclerosis.

Wnt / $\beta$-catenin pathway signaling function is to regulate cell adhesion, migration, embryo development, and tissue and organ homeostasis. [12]. In the development of the kidney, a variety of signaling pathways are involved, and abnormal activation of the Wnt/ $\beta$-catenin signaling pathway plays an important role in 
renal fibrosis. [13]. Wnt proteins can cause $\beta$-catenin degradation in the cytoplasm, leading to elevated levels of $\beta$-catenin in the nucleus and binding to the gene-specific transcription factor LEF1/TCF to induce the transcription of the target gene. This in turn induces epithelial-mesenchymal transition (EMT). In the Wnt signaling pathway, $\beta$-catenin is an important intracellular signal transductor, and several studies have confirmed that it is overactivated, eventually leading to renal fibrosis. $[14,15]$. The overactivation of this pathway is found in the glomerulus in focal segmental glomerulosclerosis and DN,too. This means that further research into the molecules that are malfunctioning in the DN process is key to developing new therapies.

Trigonelline (TRL) is an active component in the extract of fenugreek seeds. The fenugreek plant is a homologous plant of this medicine, and its pharmacological action is attributed to various components, including potato saponin, 4-hydroxy isoleucine and TRL. In the private sector, people use crude extracts of fenugreek seeds to lower blood sugar.[16]. These bioactive compounds are shown to have important pharmacological activities, such as hypoglycemic, anti-tumor, and hypolipidemic. TRL can attenuate renal fibrosis with minimal side effects and toxicity, so we primarily focused on the effects of TRL and $\beta$ catenin signaling in the evolvement of DN.

\section{Materials And Methods}

\section{Cell culture}

HMCs were provided by the American Type Culture Collection. The cells were cultured in $5.5 \mathrm{~mm} \mathrm{~d}$-glucose ( $10 \%$ thermally inactivated fetal bovine serum) and placed in a $37^{\circ}, 5 \% \mathrm{CO} 2$ incubator. The cells were sub cultured and every 2 days and divided into seven groups. Group NG was treated with normal glucose (NG, $5.5 \mathrm{mM})$, group $\mathrm{HG}$ was treated with high glucose (HG, $30 \mathrm{mM})$, group $A$ was treated with a medium dose of TRL (100 $\mu \mathrm{M}$, wkq-00259, purity > 98\%; Vic's Biological Technology Co. Ltd., Sichuan, China), group B was transfected with $\beta$-catenin siRNA and then treated with TRL $(100 \mu \mathrm{M})$, group $C$ was transfected with $\beta$-catenin pcDNA, group D was transfected with $\beta$-catenin pcDNA then treated with TRL $(100 \mu \mathrm{M})$, and group $E$ was treated with the Wnt/ $\beta$-catenin signaling pathway inhibitor ICG-001 (3 $\mu \mathrm{M} ; \mathrm{HY}-14428$, MedChemExpress Co. Ltd., New Jersey, USA).

\section{Cell viability assay}

MTT assay. Exponentially growing cells $\left(3 \times 10^{3} /\right.$ well) were plated in 96 -well plates, serum-starved overnight and grouped by NG and HG. Then divided into individual TRL concentration administration groups $(0,25,50,100,200,400$, and $800 \mu \mathrm{M})$ for different time durations $(24,48$, and $72 \mathrm{~h})$. As an osmotic control, $24.5 \mathrm{mM}$ mannitol (MA) was added to the NG medium. After the arrival time point, add each well to the MTT to a concentration of $0.5 \mathrm{mg} / \mathrm{mL}$, then place the incubator for 4 hours, discard the liquid per well, add $150 \mu \mathrm{L}$ of DMSO, wait for 10 minutes to dissolve the crystals, and measure with a microplate reader. Absorbance values. (SpectraMax Plus384; Molecular Devices, USA)

\section{Immunocytochemistry analysis}


HMCs were used as monolayer cultures and fixed in 4\% paraformaldehyde for 10-15 min. After the cells were blocked with an endogenous peroxidase blocker (ZSGB-BIO, Beijing, China) for 20 minutes. Incubation with rabbit monoclonal antibodies against $\beta$-catenin (ab32572, 1:200; Abcam, USA), Wnt5a (ab179824, 1:250; Abcam, USA) and TCF4 (ab76151, 1:200; Abcam, USA) Leave at $4{ }^{\circ} \mathrm{C}$ overnight. Then, the bound antibody was detected with horseradish peroxidase (HRP)-anti-rabbit IgG and diaminobenzidine (DAB), and then counterstained with hematoxylin. The percentage of positively stained areas in the glomeruli was analyzed by computer image.

\section{Transient transfection}

HMC was cultured in groups in a six-well plate. After the cells reached approximately $80 \%$ confluence, 2.5 $\mu \mathrm{g}$ of plasmids expressing CTNNB1 (HG11279-M-F, Sino Biological Inc., Beijing, China) were transfected into the HMCs to overexpress $\beta$-catenin using Lipofectamine 2000 reagent (11668-027, Invitrogen-BRL, Carlsbad, CA, USA) following the manufacturer's protocol. For $\beta$-catenin knockdown, 75 pmol of small interfering RNA (siRNA) against CTNNB1 (GenePharma Corporation, Shanghai, China) was transfected into the HMCs using Lipofectamine 2000 reagent. The siRNA sequences are as follows: si-CTNNB1 (Human) sense 5'- GGGUUCAGAUGAUAUAAAUTT-3' and antisense 5'-AUUAUAUCAUCUGAACCCAG-3'. The siRNA/liposome and pcDNA/liposome complexes were added to the serum-free medium and the complexes were formed at room temperature for $20 \mathrm{~min}$. After $4 \mathrm{~h}$ of incubation, the medium containing HG (30 mM) was added and incubation continued. After 48, extracting each group of cellular proteins.

\section{PCR analysis}

TRIzol Reagent (15596026, Invitrogen, USA). PCR was executed on the 7500 FAST Real-Time PCR System for 45 cycles(Applied Biosystems, Carlsbad, CA). The thermocycling conditions were as follows: Initial melting at $94^{\circ} \mathrm{C}$ for $30 \mathrm{sec}$ followed by 45 cycles of $94^{\circ} \mathrm{C}$ for $5 \mathrm{sec}, 51^{\circ} \mathrm{C}$ for $15 \mathrm{sec}$, and $72^{\circ} \mathrm{C}$ The $10 \% \mathrm{sec}$. The GAPDH mRNA was amplified at the same time and used as a loading control. The relative amounts of mRNA were determined by $2-\Delta \Delta \mathrm{Cq}$ method. Each sample was tested in triplicate. Primer sequences are shown in Table 1. 
Table 1

Sequences of specific primers for qRT-PCR

\begin{tabular}{|c|c|c|}
\hline Genes & Primers & Product Length \\
\hline \multirow[t]{4}{*}{ CTNNB1 } & Forward & \multirow[t]{4}{*}{$211 b p$} \\
\hline & 5'-ACAGGGAAGACATCACTGAGCCTGC-3' & \\
\hline & Reverse & \\
\hline & 5'-GGTGCATGATTTGCGGGACAAAG-3' & \\
\hline \multirow[t]{2}{*}{ Wnt5a } & Forward 5'- GGCTGGAAGTGCAATGTCTT-3' & \multirow[t]{2}{*}{$289 b p$} \\
\hline & Reverse 5'- CCTTCGATGTCGGAATTGAT-3' & \\
\hline \multirow[t]{2}{*}{ TCF4 } & Forward 5'-CCAATCACGACAGGAGGATT-3' & \multirow[t]{2}{*}{$298 b p$} \\
\hline & Reverse 5'-CGACCTTTGCTCTCATTTCC-3' & \\
\hline \multirow[t]{2}{*}{ Cyclin D1 } & Forward 5'- GCTGCGAAGTGGAAACCATC-3' & \multirow[t]{2}{*}{$135 \mathrm{bp}$} \\
\hline & Reverse 5'-СCTСCTTCTGCACACATTTGAA-3' & \\
\hline \multirow[t]{2}{*}{ CDK4 } & Forward 5'- ATGGCTACCTCTCGATATGAGC-3' & \multirow[t]{2}{*}{$124 b p$} \\
\hline & Reverse 5'-CATTGGGGACTCTCACACTCT-3' & \\
\hline \multirow[t]{2}{*}{ GAPDH } & Forward 5'- CAAGGTCATCCATGACAACTTTG-3' & \multirow[t]{2}{*}{$496 b p$} \\
\hline & Reverse 5'- GTCCACCACCCTGTTGCTGTAG-3' & \\
\hline
\end{tabular}

Total Protein Extraction Kit (SD-001, Invent Biotechnologies Inc., USA). An equal amount of protein was loaded onto a $12 \%$ sodium dodecyl sulfate-polyacrylamide gel for separation. The protein was then transferred to a polyvinylidene fluoride membrane (Merck Millipore, MA, USA) and blocked with 5\% skim milk for 1 hour. Incubate the membrane and primary antibodies overnight at $4^{\circ} \mathrm{C}$ The next day, HRPconjugated anti-rabbit IgG secondary antibody (ZB-2301, 1:3000; ZSGB-Bio) was incubated for 1 hour. The Tanon 4200 Automated Chemiluminescence Image Analysis System (Tanon, Shanghai, China) took photos. Image-Pro Plus 6.0 for image analysis.

\section{Cell cycle}

The HMC was inoculated to a 6 -well plate with a density of $1 \times 104$.After 48 hours, PBS was washed twice and collected, fixed in $70 \%$ cold ethanol at $4{ }^{\circ} \mathrm{C}$ overnight. Next, the fixed cells were resuspended at room temperature with $50 \mathrm{~g} / \mathrm{ml}$ propiridine iodide (PI).CF0031, Beijing dingguo biotechnology co., LTD.) binding buffer in 30 minutes. Then flow cytometry for detection. (Becton Dickinson, SAN jose, ca, USA) ModFit LT V3.3.11 (Verity Software House Inc., topsom, Maine, USA) was used to determine the proportion of G0 / G1, S and G2 / M cells. 


\section{Cell apoptosis analysis}

Annexin v-fitc /PI kit detecte cell apoptosis.(ca1020-50, Solarbio Science \& Technology Co. Ltd., Beijing, China) Cells were washed with pre-cooled PBS and collected, then fixed at $4^{\circ} \mathrm{C}$ overnight in $70 \%$ ethanol.After 72 hours of incubation, cells were collected and centrifuged at $4^{\circ} \mathrm{C}$ and $100 \mathrm{~g}$ for 5 minutes, gently aspirate the supernatant and mix well with $1 \mathrm{ml}$ PBS. Annexin V - FITC and PI ( 5 uL each) were then added to The tubes, mixed gently, and incubated for $15 \mathrm{~min}$. Then,300 mul of binding buffer added and apoptosis was detected within $1 \mathrm{~h}$.

\section{TUNEL analysis}

The TUNEL apoptosis detection kit was used in this experiment (C1089, Beyotime Institute of Biotechnology, Shanghai, China).DNA strand breakage was evaluated by fluorescence labeling of the terminal dUTP notch (TUNEL).DAPI solution (c02-04002, Bioss Biotechnology Co. Ltd., Beijing, China) was used on nuclear staining. TUNEL positive cells were counted in the defined area and their density was calculated.

\section{Statistical analysis}

Data are presented as mean \pm SEM with $n$ denoting the sample size in each group. Differences between groups were analyzed by variance analysis or rank sum test. One-way ANOVA tests were used to compare three or more independent groups. Statistical analysis was performed using SPSS software version 19.0. $\mathrm{P}<0.05$ was considered statistically significant.

\section{Results}

TRL suppressed HG-induced excessive proliferation of HMCs in a concentration-dependent manner.

To examine the effect of TRL on HG-induced HMCs, HMCs were cultured and the MTT assay was used to detect cell proliferation for different periods of time $(24,48$, and $72 \mathrm{~h})$. Cells cultured under HG conditions for $24 \mathrm{~h}$ proliferated faster than the untreated cells $(P<0.01)$ and the difference was still significant after $48 \mathrm{~h}(\mathrm{P}<0.01)$. However, the proliferation rate of HG-treated cells decreased after $72 \mathrm{~h}$. Treatment with mannitol did not alter cell growth at any of the time points tested, suggesting that the HG-induced HMC proliferation was not due to high osmotic pressure. Compared to the proliferation rate in the HG group, that in the cells treated with TRL at a concentration of 25 to $800 \mu \mathrm{M}$ decreased significantly $(P<0.05)$, and the effect was concentration-dependent (Fig. 1). The results show that HG can effectively promote the proliferation of HMCs in a time-dependent manner, and this effect was most significant at $48 \mathrm{~h}$. TRL suppressed the HG-induced excessive proliferation of HMCs in a concentration- and time-dependent manners for the first $48 \mathrm{~h}$. Therefore, we chose the $48 \mathrm{~h}$ time point for further experiments, including cellcycle, western blot, RT-PCR, and immunocytochemistry analyses. We chose the $72 \mathrm{~h}$ time point for the apoptotic analyses.

TRL down-regulated Wnt/ $\beta$-catenin signaling in HMCs under HG conditions. 
To assess whether the effects of TRL on cell proliferation were associated with the Wnt/ $\beta$-catenin signaling pathway, western blot and RT-PCR analyses were performed. We tested three concentrations of $\operatorname{TRL}(50,100$, and $200 \mu \mathrm{M})$ for these experiments to choose the optimum concentration. As shown in Fig. $2 \mathrm{C}$, the protein and mRNA expression of $\beta$-catenin, Wnt5a, and TCF4 were significantly increased following HG treatment, however they were inhibited by TRL in a dose-dependent manner. TRL reversed the increase in the expression of $\beta$-catenin, Wnt5a, and TCF4, with the most significant response observed at the $100 \mu \mathrm{M}$ concentration. Therefore, we used $100 \mu \mathrm{M}$ TRL in all subsequent experiments. The results of the immunohistochemistry staining show that the expression of $\beta$-catenin, Wnt5a, and TCF4 is higher in the HG group than that in the NG group. Of these, the staining for $\beta$-catenin was positive. We observed that HG promoted $\beta$-catenin entry into the nucleus and formation of a transcriptional complex with TCF4. Compared to the HG group, the number of positive cells in the TRL-treated group significantly decreased (Fig. 2B). These results suggest that TRL inhibited the HG-induced HMC proliferation by inhibiting the Wnt/ $\beta$-catenin signaling pathway.

Signaling mechanism for the anti-diabetic property of TRL in HMCs.

The above results demonstrate the beneficial effects of TRL against HG-induced over-proliferation of HMCs. To shed light on how TRL mediates these effects, we use the Wnt/ $\beta$-catenin pathway inhibitor ICG001 and studied the expression of $\beta$-catenin, Wnt5a, and TCF4. We found that TRL had the same effects on these signals as ICG-001. These results indicate that TRL might influence the survival of HMCs by the inhibition of the Wnt pathway. Next, we chose to transiently transfect HMCs with plasmids expressing $\beta$ catenin. We found that the signals of the Wnt/ $\beta$-catenin pathway increased significantly, but decreased when treated with TRL at the same time. These results revealed that HG might lead to an increase in $\beta$ catenin. Additionally, TRL was able to reduce the upregulation of both the mRNA and protein levels of $\beta$ catenin. Compared with that observed for the HG group, the expression of each signal factor was significantly down-regulated in the $100 \mu \mathrm{M}$ of TRL and the $\beta$-catenin siRNA group (group B; $P<0.05$ ), and the expression level was also significantly reduced compared to that of group A treated with TRL alone (Fig. 3). These results indicate that TRL mediates its effects when the Wnt signaling is active and that TRL not only down-regulates the high expression of $\beta$-catenin but also has a significant regulatory effect on the other factors in the Wnt/ $\beta$-catenin signaling pathway.

TRL suppressed HG-induced cell-cycle progression in HMCs.

To further examine the role of TRL in HMC cell-cycle progression, flow cytometry analysis was performed. As shown in Fig. 4A, $H G$ induced a decrease in the percentage of cells in the $G_{1}$ phase but increased those in the $S$ phase, indicating that $H G$ promoted cell cycle progression. In contrast, TRL arrested cells in the $G_{1}$ phase $(p<0.05)$, and therefore, the number of cells in the $S$ phase was significantly lower $(p<0.05)$. After treatment with siRNA and TRL (group B), the lowest proportion of cells was in the S phase. The ICG001 intervention (group E) also had a definite block in the $S$ phase. The acceleration of the cell cycle in the $\beta$-catenin overexpression group (group $C$ ) was more significant than that in the HG group, and the percentage of cells in the $S$ phase for group $D$ was significantly reduced after treatment with TRL $(P<$ 
0.05). High expression of Cyclin D1 and CDK4 was observed in group HG and group C, while TRL significantly decreased the expression of Cyclin D1 and CDK4 (Fig. 4B and C). These results suggest that HG could promote cell proliferation by speeding up the cell-cycle during the first $48 \mathrm{~h}$, but TRL could block the $\mathrm{HG}$-induced cell cycle in $\mathrm{G}_{1} / \mathrm{S}$-phase.

TRL reduced HMCs apoptosis via the Wnt/ $\beta$-catenin pathway.

To examine whether TRL and the regulation of Wnt/ $\beta$-catenin signaling effects the apoptosis of HMCs, flow cytometry and TUNEL assays were performed. Compared to the NG group, the apoptosis rate was significantly increased in the HG group $(P<0.05)$. Additionally, the apoptosis rate increased more significantly following transfection with $\beta$-catenin pcDNA $(P<0.01)$. TRL could reduce the apoptosis induced by $\beta$-catenin pcDNA. The rate of early apoptosis observed following the treatment with TRL alone (group A), and in combination with the silencing of $\beta$-catenin (group B) was significantly lower than that of group HG $(P<0.05)$. There was a significant difference between groups $B$ and $A(P<0.05)$. The apoptosis of HMCs after ICG-001 intervention (group E) also decreased significantly $(P<0.05 ;$ Fig. 5A). This is consistent with the results of the TUNEL assay. TUNEL staining indicated that HG and the overexpression of $\beta$-catenin induced DNA fragmentation, a sign of apoptosis. Under HG conditions, HMCs displayed DNA fragmentation, however only a few cells treated with TRL showed DNA fragmentation. DAPI staining showed that some nuclei had a rounded shape. Interestingly, group C had more DNA fragmentation than the HG group (Fig. 5B). This difference may arise from the differential expression of $\beta$-catenin. Both results indicated that HG could lead to cell apoptosis after $72 \mathrm{~h}$. The expression of $\beta$ catenin might be related to the apoptosis of HMCs; its accumulation in the cytoplasm may to a certain extent lead to apoptosis. However, TRL as well as the regulation of $\beta$-catenin could be attributed to the reduction of cell apoptosis.

\section{Discussion}

DN is a glomerular sclerosis disease caused by the abnormal metabolism of diabetes mellitus. High glucose easily affects mesangial cells, which in turn cause cellular stress and damage [17]. The proliferation and hypertrophy of mesangial cells as well as the accumulation of an extracellular matrix eventually led to glomerular sclerosis. HG was recognized not only as the main characteristic of DN but also as a risk factor for the occurrence and development of DN [18]. Research confirmed HG can promote proliferation of HMCs [19-21]. Our study provides evidence that TRL can inhibit HG-induced HMC overproliferation in a concentration-dependent manner. HG causes mesangial cell damage because HG activates a molecular pathway leading to apoptosis. HG reduces the survival rate of glomerular cells in a variety of ways, such as increasing oxidative stress or altering growth factor expression [22, 23]. To our knowledge, the biological effects of TRL in measuring the survival of HMCs under HG conditions are not clearly defined. Therefore, TRL's effect in inhibiting proliferation of HMCs might become an effective treatment for the prevention and treatment of DN. 
The development of DN is associated with the abnormal proliferation of mesangial cells, and HG conditions could induce proliferation of mesangial cells. In this experiment, the effect of TRL on the proliferation of HMCs was studied by MTT assays. It was found that the absorbance increased significantly in the HG group at $48 \mathrm{~h}$, suggesting that hyperglycemia stimulated excessive proliferation of HMCs within the experimental dose range. TRL could inhibit the HMCs hyperproliferation induced by HG in a concentration-dependent manner. After the HG treated cells were cultured for $72 \mathrm{~h}$, cell proliferation slowed and cell viability significantly decreased. However, TRL could enhance HMC activity at this time. Since HG conditions had the most significant effect on the proliferation of HMCs at $48 \mathrm{~h}$, this time point was selected to further study the effect of TRL on the proliferation of HMCs under HG and to understand its mechanism.

There are three phases of the cell cycle: G0/G1 phase, DNA synthesis-related S phase, and G2/M phase. $[24,25]$. Cyclin D1 is a cell cycle regulator of CDK4, and it acts mainly in the $G_{1}$ phase. The active complexes formed by their combination can promote cells to enter the $S$ phase from the $G_{1}$ phase. The $S$ phase is the DNA replication phase. Once the cells enter the $S$ phase, cell division can proceed until the next $G_{1}$ phase. The HMCs proliferated at low rates under normal glucose conditions and largely remained in the $G_{0} / G_{1}$ phase of the cell cycle. Since $H G$ stimulation, cells in the $G 0$ / G1 phase are reduced, and the increase in the number of the $S$ and G2 / M phase. TRL could significantly reverse these effects. These results demonstrate that $\mathrm{HG}$ mainly played a role of promoting proliferation, but TRL might reduce the $\mathrm{HG}$-induced over-proliferation of HMCs by inhibiting their transition from the $G_{0} / G_{1}$ to $S$ phase. To explore whether there is a relationship between cell proliferation and the $\mathrm{Wnt} / \beta$-catenin pathway, we transiently over-expressed $\beta$-catenin. We found that the cell cycle in group $C$ speeded up significantly, whereas the group D which was slowed by TRL had the opposite effect. High expression of Cyclin D1 and CDK4 was observed in group $\mathrm{HG}$ and group $\mathrm{C}$, while TRL significantly decreased their expression. In view of the results above, we concluded that HG conditions and high expression of $\beta$-catenin could accelerate the cell cycle and promote cell proliferation, while TRL could down-regulate the expression of Cyclin D1 and CDK4 and regulate $G_{1} / S$ phase monitoring to cause $G_{1}$ arrest. Thus, slowing down the cell cycle. TRL and the Wnt/ $\beta$-catenin pathways might participate in the process of the cell cycle.

The Wnt / $\beta$-catenin signaling pathway is a classic entry point for diabetic nephropathy. Sustained activation of the $W n t / \beta$-catenin signaling pathway is commonly associated with proteinuria and glomerulosclerosis in $D N[26,27]$. Since $\beta$-catenin is an important regulatory factor in the $\mathrm{Wnt} / \beta$-catenin signaling pathway, we used pcDNA3.1- $\beta$-catenin and siRNA transfected cells to overexpress or knock down $\beta$-catenin, respectively, to probe the HG conditions in this study. Then we detected the expression levels of $\beta$-catenin, Wnt5a, and TCF4 by western blotting and qRT-PCR. After transient transfection of plasmids into HMCs, the signal intensity of the Wnt/ $\beta$-catenin signaling pathway significantly increased, but the expression of related factors in the Wnt/ $\beta$-catenin signaling pathway was significantly reduced after the $100 \mu \mathrm{M}$ TRL intervention. These results demonstrate that HG conditions can activate the Wnt/ $\beta$ catenin signaling pathway, leading to an increase in $\beta$-catenin expression. Additionally, TRL could downregulate the expression of $\beta$-catenin mRNA and protein levels and inhibit the Wnt/ $\beta$-catenin pathway. The

Page 10/19 
expression of Wnt5a, $\beta$-catenin, and TCF4 was significantly reduced by the intervention with TRL alone, and the expression of these factors was also reduced after knockdown of $\beta$-catenin and the TRL intervention at the same time. This suggests that TRL not only down-regulates the high expression of $\beta$ catenin but also regulates other factors in the Wnt/ $\beta$-catenin signaling pathway.

There are many reasons why HG promotes apoptosis of mesangial cells, mainly due to changes in growth factor expression or increased oxidative stress. [28-30]. Instead, the biologic role of the Wnt/ $\beta$ catenin pathway in measuring the apoptosis of HMCs that are exposed to HG has not been extensively studied. $\beta$-catenin signaling is involved in many cell processes, for examples: cell proliferation, differentiation, and apoptosis. Recently research, we examined the effect of TRL on the sustained effect of Wnt/ $\beta$-catenin signaling and its effect on reducing apoptosis in HG-stressed mesangial cells. We found that the percentage of apoptotic cells slightly increased under $\mathrm{HG}$ conditions after $72 \mathrm{~h}$. The apoptosis rate significantly increased in group $C$, in which $\beta$-catenin was overexpressed. This implies that the high expression of $\beta$-catenin might lead to cell apoptosis. These results imply that apoptosis-related molecules are modulated by regulating the canonical Wnt/ $\beta$-catenin signaling pathway, which is caused by HG induction of HMCs apoptosis. The knock-down in $\beta$-catenin levels following treatment of HMCs with TRL might account for the survival of HG-stressed cells.

In short, results demonstrated that $\mathrm{HG}$ condition and $\beta$-catenin overexpression could activate the Wnt/ $\beta$ catenin signaling pathway, while TRL inhibited Wnt/ $\beta$-catenin signaling by regulating the expression of $\beta$ catenin, TCF4, and other related factors. TRL could down-regulate the expression of Cyclin D1 and CDK4, stopping the cell cycle at the $\mathrm{G}_{1}$ phase. Inhibition of abnormal proliferation is induced by $\mathrm{HG}$ and overexpression of $\beta$-catenin. TRL inhibited apoptosis by down-regulating $\beta$-catenin signaling. This provides a novel insight into the understanding of the molecular and signaling pathways through which TRL suppresses HG-induced cell over-proliferation and apoptosis. Thus, treatment with TRL as well as the modulation of the Wnt/ $\beta$-catenin pathway represent novel therapeutic strategies for the treatment of DN in the clinical setting.

\section{Declarations}

\section{Ethics approval and consent to participate}

Not applicable.

\section{Consent for publication}

Not applicable.

\section{Data Availability}

The data used to support the findings of this study are included within the article. 
The authors declare that they have no competing interests.

\section{Funding:}

This work was supported by funding from the Jilin Province Science and Technology Department (No.20190701045GH, No.20190201086JC).

\section{Author Contributions}

C.C. and Y.Z. conceived and designed the experiments. C.C., Y.S. and Z.C. performed the experiments. X.L. and B.S. analyzed data. X.Y., J.M., H.Z. and X.R. contributed to the preparation of reagents/materials/analysis tools. C.C. wrote the manuscript. Y.Z. supervised this study.

Acknowledgments: We thank Editage ( https://www.editage.com/frontiers/ ) for its linguistic assistance after the preparation of this manuscript.

\section{References}

1. Kowalski A, Krikorian A, Lerma EV. Diabetes and chronic kidney disease. Dis Mon. 2015;61(9):37886.

2. Sharma D, et al. Diabetic nephropathy: New insights into established therapeutic paradigms and novel molecular targets. Diabetes Res Clin Pract. 2017;128:91-108.

3. Magee C, et al. Diabetic Nephropathy: a Tangled Web to Unweave. Cardiovasc Drugs Ther; 2017.

4. Ostojic $P$, Stojanovski N. Arterial hypertension treated with angiotensin converting enzyme inhibitors and glucocorticoids are independent risk factors associated with decreased glomerular filtration rate in systemic sclerosis. Rheumatol Int. 2017;37(3):363-8.

5. Reidy K, et al. Molecular mechanisms of diabetic kidney disease. J Clin Invest. 2014;124(6):233340.

6. Badal SS, Danesh FR. New insights into molecular mechanisms of diabetic kidney disease. Am J Kidney Dis. 2014;63(2 Suppl 2):S63-83.

7. Kanwar YS, et al. A Glimpse of Various Pathogenetic Mechanisms of Diabetic Nephropathy. Annu Rev Pathol. 2011;6:395-423., ,. 6.

8. Wang J, et al. Effects of Free Anthraquinones Extract from the Rhubarb on Cell Proliferation and Accumulation of Extracellular Matrix in High Glucose Cultured-Mesangial Cells. Korean J Physiol Pharmacol. 2015;19(6):485-9.

9. Kim MY, et al. Resveratrol prevents renal lipotoxicity and inhibits mesangial cell glucotoxicity in a manner dependent on the AMPK-SIRT1-PGC1alpha axis in db/db mice. Diabetologia. 2013;56(1):204-17.

10. Jardim DP, Poco PCE, Campos AH. Dact1, a Wnt-Pathway Inhibitor, Mediates Human Mesangial Cell TGF-beta1-Induced Apoptosis. J Cell Physiol. 2017;232(8):2104-11. 
11. Yang SM, et al. Osthole improves an accelerated focal segmental glomerulosclerosis model in the early stage by activating the Nrf2 antioxidant pathway and subsequently inhibiting NF-kappaBmediated COX-2 expression and apoptosis. Free Radic Biol Med. 2014;73:260-9.

12. Kawakami T, Ren S, Duffield JS. Wnt signalling in kidney diseases: dual roles in renal injury and repair. J Pathol. 2013;229(2):221-31.

13. Edeling $\mathrm{M}$, et al. Developmental signalling pathways in renal fibrosis: the roles of Notch, Wnt and Hedgehog. Nat Rev Nephrol. 2016;12(7):426-39.

14. Tan RJ, et al, Wnt/beta-catenin signaling and kidney fibrosis. Kidney Int Suppl (2011), 2014. 4(1): p. 84-90.

15. Madan B, et al. Experimental inhibition of porcupine-mediated Wnt O-acylation attenuates kidney fibrosis. Kidney Int. 2016;89(5):1062-74.

16. Aldakinah AA, et al. Trigonelline and vildagliptin antidiabetic effect: improvement of insulin signalling pathway. J Pharm Pharmacol. 2017;69(7):856-64.

17. Bhattacharjee $\mathrm{N}$, et al. Mechanistic insight of diabetic nephropathy and its pharmacotherapeutic targets: An update. Eur J Pharmacol. 2016;791:8-24.

18. Giacco F, et al. Knockdown of glyoxalase 1 mimics diabetic nephropathy in nondiabetic mice. Diabetes. 2014;63(1):291-9.

19. Lin CL, et al. Simvastatin reverses high glucose-induced apoptosis of mesangial cells via modulation of Wnt signaling pathway. Am J Nephrol. 2008;28(2):290-7.

20. Zeng R, et al. Fenofibrate attenuated glucose-induced mesangial cells proliferation and extracellular matrix synthesis via PI3K/AKT and ERK1/2. PLoS One. 2013;8(10):e76836.

21. Song SE, et al. Delphinidin prevents high glucose-induced cell proliferation and collagen synthesis by inhibition of NOX-1 and mitochondrial superoxide in mesangial cells. J Pharmacol Sci. 2016;130(4):235-43.

22. Xu L, et al. Inhibition of autophagy increased AGE/ROS-mediated apoptosis in mesangial cells. 7: Cell Death \& Disease; 2016.

23. Ho C, et al. Curcumin Rescues Diabetic Renal Fibrosis by Targeting Superoxide-Mediated Wnt Signaling Pathways. Am J Med Sci. 2016;351(3):286-95.

24. Tian R, Li Y, Gao M. Shikonin causes cell-cycle arrest and induces apoptosis by regulating the EGFRNF-kappaB signalling pathway in human epidermoid carcinoma A431 cells. Biosci Rep, 2015. 35(2).

25. Kim SJ. Berberine inhibits proliferation by cell cycle arrest at the G2/M phase via PI3K /Akt and p38 kinase in HTB-94 human chondrosarcoma cell line. Febs Journal. 2015;282:284-4.

26. Hu C, et al. Insights into the Mechanisms Involved in the Expression and Regulation of Extracellular Matrix Proteins in Diabetic Nephropathy. Curr Med Chem. 2015;22(24):2858-70.

27. Guo J, et al. VDR Activation Reduces Proteinuria and High-Glucose-Induced Injury of Kidneys and Podocytes by Regulating Wnt Signaling Pathway. Cell Physiol Biochem. 2017;43(1):39-51.

28. Khera T, et al. Glucose enhances mesangial cell apoptosis. Lab Invest. 2006;86(6):566-77. 
29. Carrillo-Lopez N, et al. Direct inhibition of osteoblastic Wnt pathway by fibroblast growth factor 23 contributes to bone loss in chronic kidney disease. Kidney Int. 2016;90(1):77-89.

30. Hsu YC, et al. Nitric oxide donors rescue diabetic nephropathy through oxidative-stress- and nitrosative-stress-mediated Wnt signaling pathways. Journal of Diabetes Investigation. 2015;6(1):24-34.

\section{Figures}

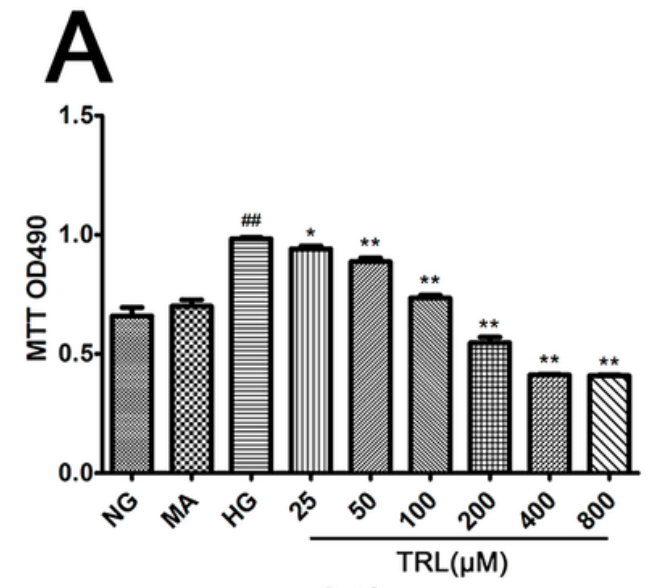

$24 \mathrm{~h}$

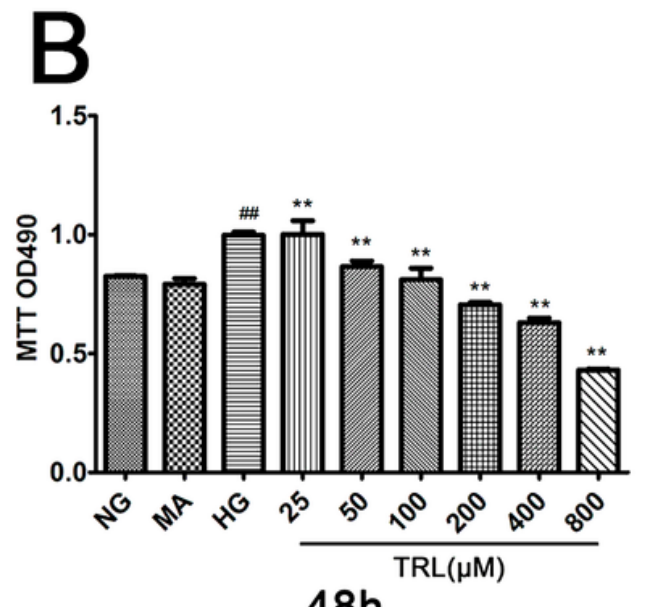

$48 \mathrm{~h}$

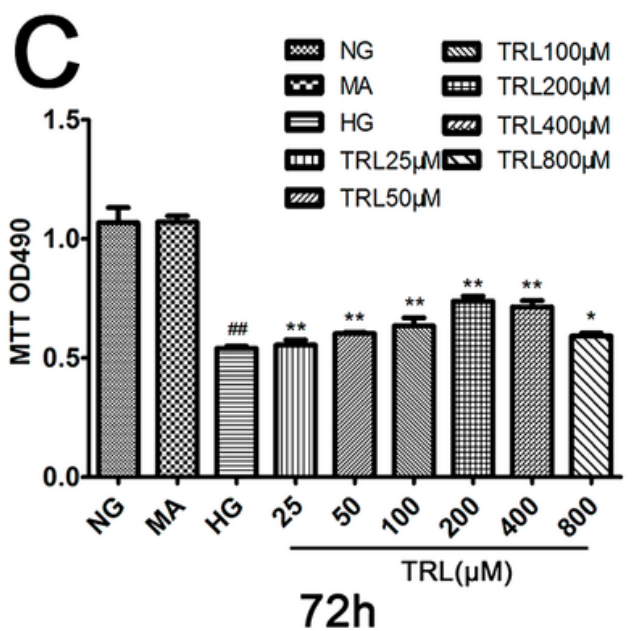

$72 \mathrm{~h}$

Figure 1

Growth-inhibitory effects of TRL on HMCs under the high-glucose conditions. Cell viability was measured by an MTT assay. Cells were treated with various concentrations of TRL $(25-800 \mu \mathrm{M})$ for $24 \mathrm{~h}(\mathrm{~A}), 48 \mathrm{~h}$ (B), and $72 \mathrm{~h}(\mathrm{C})$; mannitol (24.5 mM, MA) was used as an osmotic control. The data are presented as the mean $\pm S E M$ from at least three independent experiments. NG: normal glucose, HG: high glucose, MA: mannitol; $\# P<0.05$ versus $N G, \# \# P<0.01$ versus NG; $* P<0.05$ versus $H G, * \star P<0.01$ versus $H G$. 


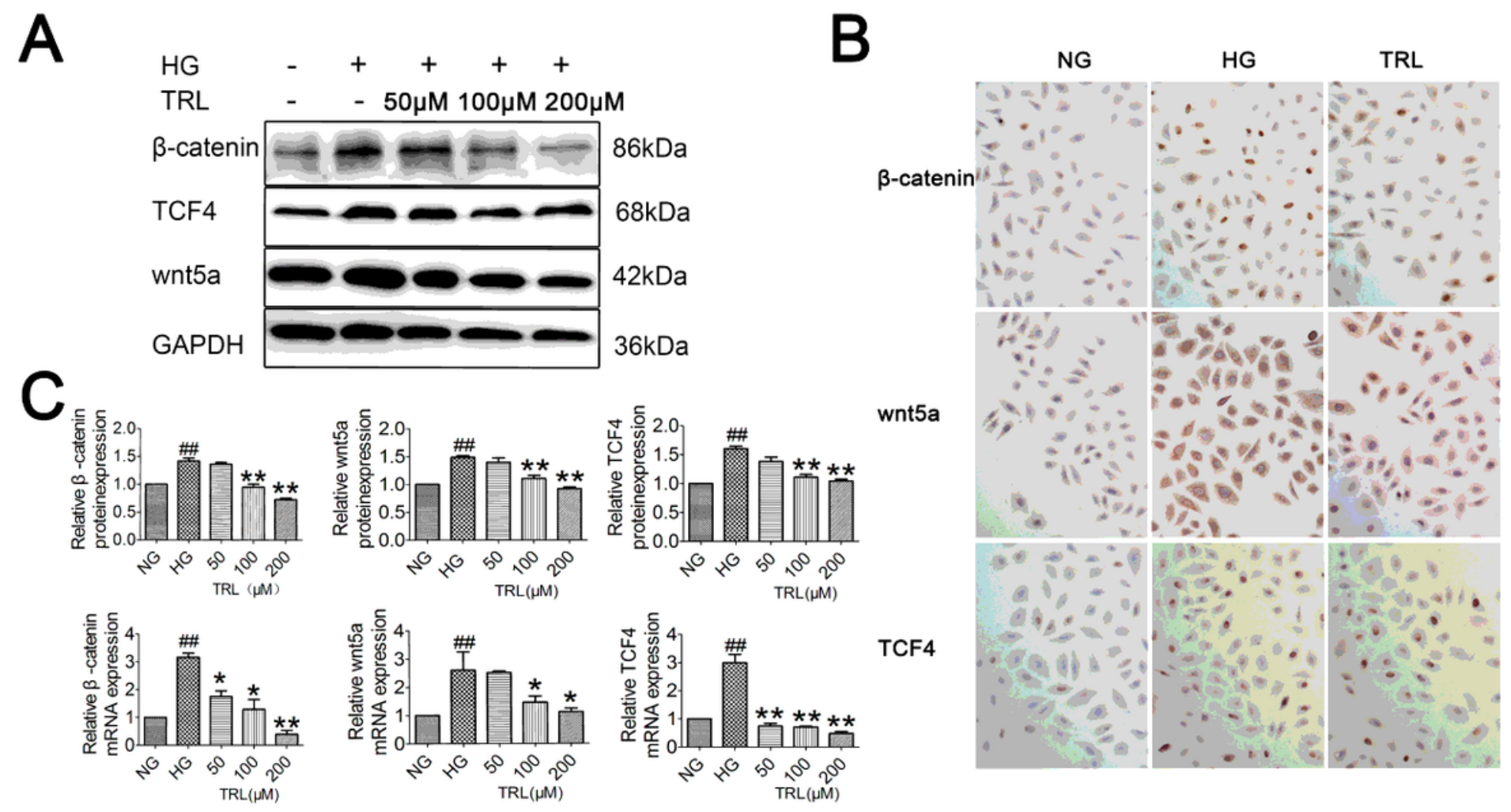

Figure 2

Effects of TRL on $\beta$-catenin, Wnt5a, and TCF4 expression in HMCs. HMCs were treated with various concentrations of TRL $(50,100$, or $200 \mu \mathrm{M})$ for $24 \mathrm{~h}$. Proteins were extracted from cells and assessed by western blot analysis. Total RNA was extracted from treated cells and assessed by qRT-PCR analysis. GAPDH served as the loading control. (A) Representative western blots of $\beta$-catenin, Wnt5a, and TCF4 expression. (B) Immunohistochemical staining (200x) of $\beta$-catenin, Wnt5a, and TCF4. (C) $\beta$-catenin, Wnt5a, and TCF4 protein and mRNA expression were detected by western blot and qRT-PCR, respectively. NG: normal glucose, HG: high glucose; \#P<0.05 versus NG, \#\#P<0.01 versus NG; * $P<0.05$ versus $H G$, ${ }^{*} \mathrm{P}<0.01$ versus $\mathrm{HG}$. 
A

$\begin{array}{lccccccc}\text { HG } & - & + & + & + & + & + & + \\ \text { TRL } & - & - & + & + & - & + & - \\ \text { SiRNA } & - & - & - & + & - & - & - \\ \text { pCDNA } & - & - & - & - & + & + & - \\ \text { ICG-001 } & - & - & - & - & - & - & +\end{array}$
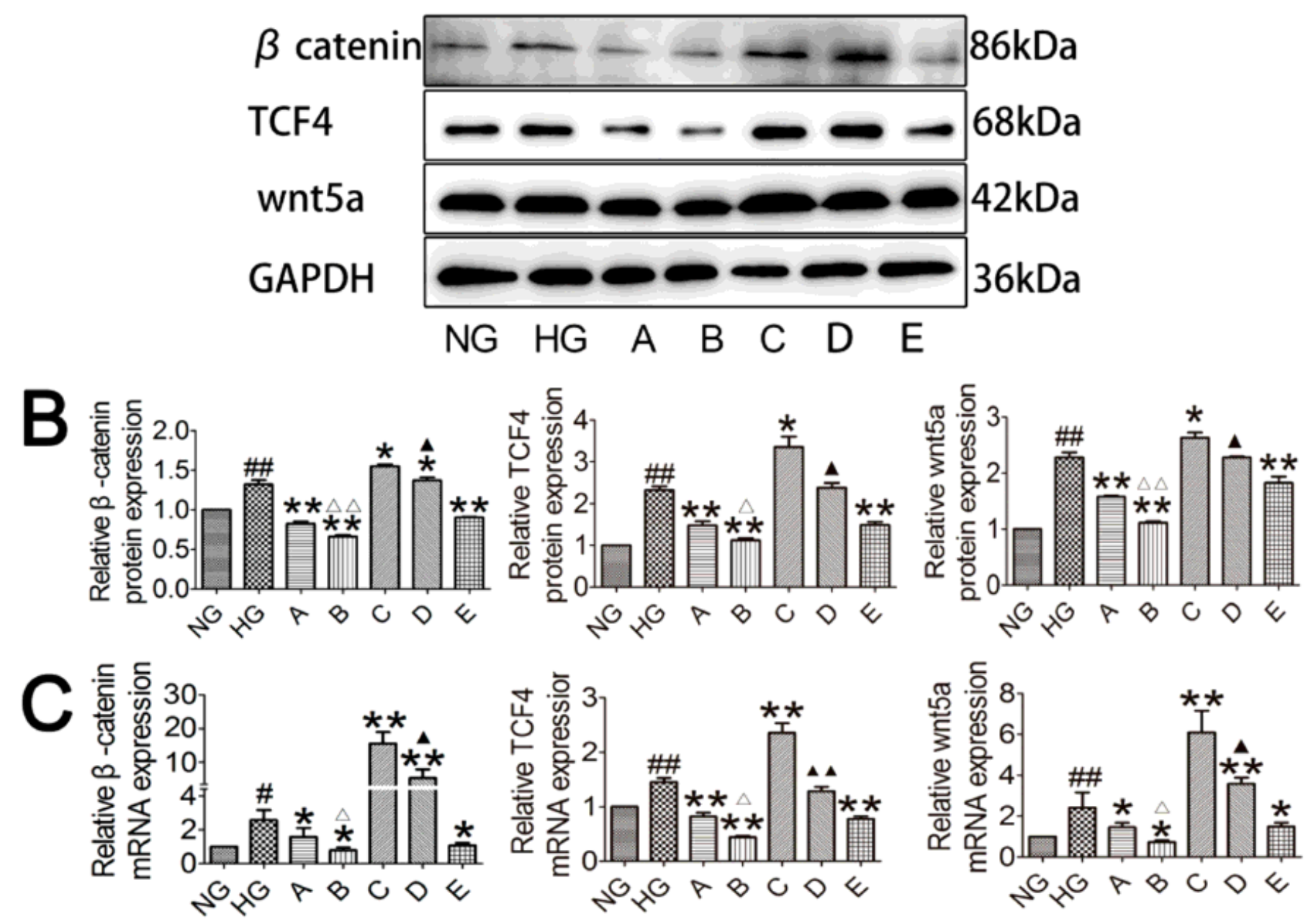

Figure 3

Expression of $\beta$-catenin, Wnt5a, and TCF4 in HMCs. After treatment, proteins were extracted from the cells and assessed by western blot analysis. Total RNA was extracted from treated cells and assessed by qRTPCR analysis. GAPDH served as the loading control. (A) Representative western blot of $\beta$-catenin, Wnt5a, and TCF4 expression. (B, C) The relative $\beta$-catenin, TCF4, and Wnt5a protein and mRNA expression detected by western blot and qRT-PCR, respectively. NG: normal glucose, HG: high glucose, $A$ : TRL, B: TRL+ $\beta$-catenin siRNA, C: $\beta$-catenin pcDNA, D: TRL+ $\beta$-catenin pcDNA, E: ICG-001; \#P<0.05 versus NG, \#\#P<0.01 versus $N G$; ${ }^{*} P<0.05$ versus $H G$, ${ }^{*} P<0.01$ versus $H G ; \triangle P<0.01$ versus group $A, \triangle \triangle P<0.01$ versus group $A ; \Delta P<0.05$ versus group $C, \Delta \Delta P<0.01$ versus group $C$. 

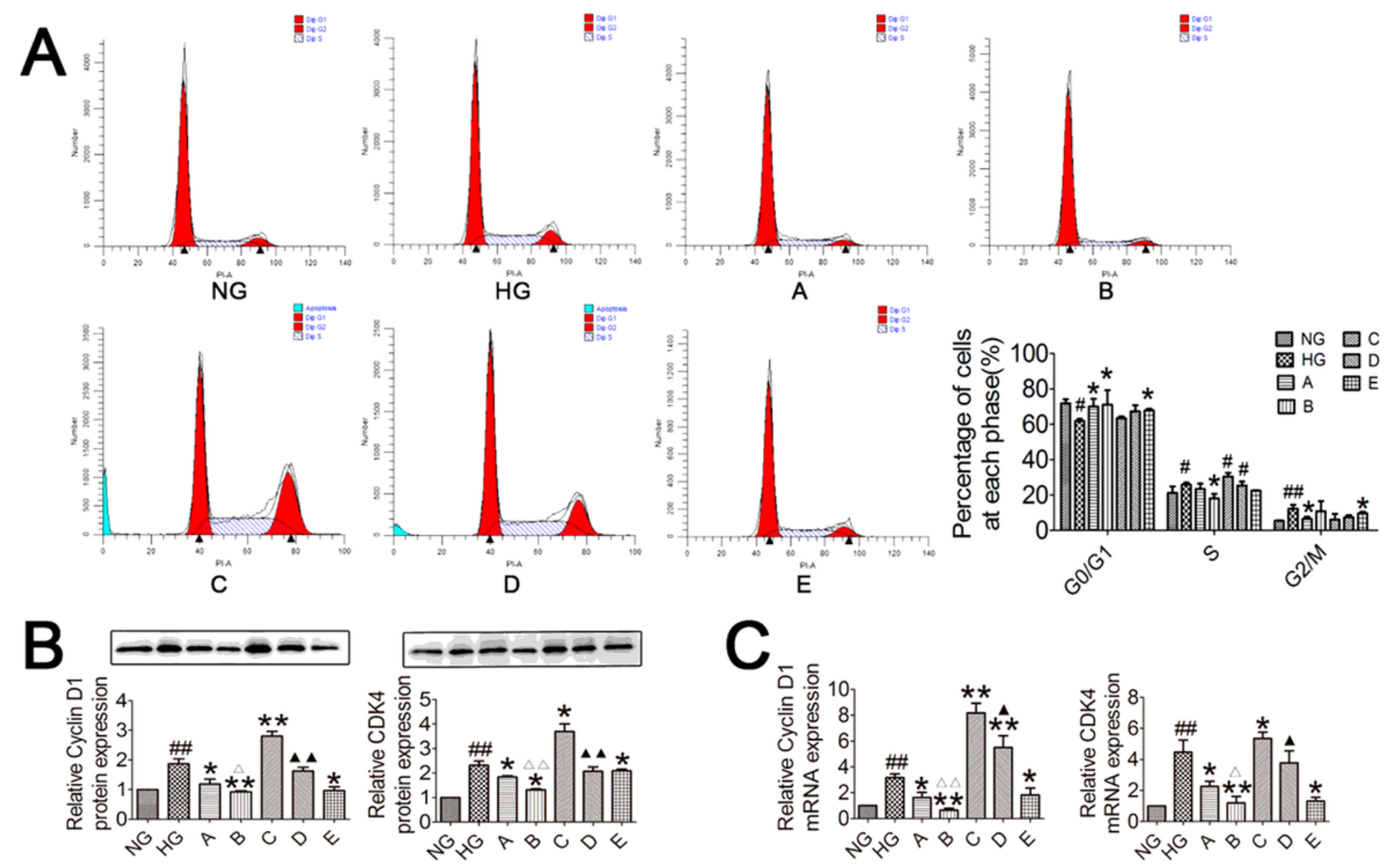

Figure 4

Effects of TRL on the cell cycle of HMCs induced by high glucose (A). Effects of TRL on Cyclin D1 and CDK4 protein and mRNA expression in HMCs $(B, C)$. The cell cycle distribution was determined by assessing the individual nuclear DNA content reflected by the fluorescence intensity of incorporated $\mathrm{PI}$. Flow cytometry analyzed the percentages of cells in $\mathrm{G0} / \mathrm{G} 1, \mathrm{~S}$, and G2/M phases after the indicated treatments. Representative flow cytometry graphs are shown and the results are presented as the mean $\pm S E M$ from at least three independent experiments. NG: normal glucose, $H G$ : high glucose, $A: T R L$, B: TRL $+\beta$-catenin siRNA, C: $\beta$-catenin pcDNA, D: TRL $+\beta$-catenin pcDNA, E: ICG-001; \#P<0.05 versus NG, \#\#P<0.01 versus NG; ${ }^{*} P<0.05$ versus $H G$, ${ }^{\star *} P<0.01$ versus $H G, \triangle P<0.05$ versus $A, \triangle \triangle P<0.01$ versus $A$; $\Delta P<0.05$ versus $C, \boldsymbol{\Delta} \mathbf{\Delta} P<0.01$ versus $C$. 

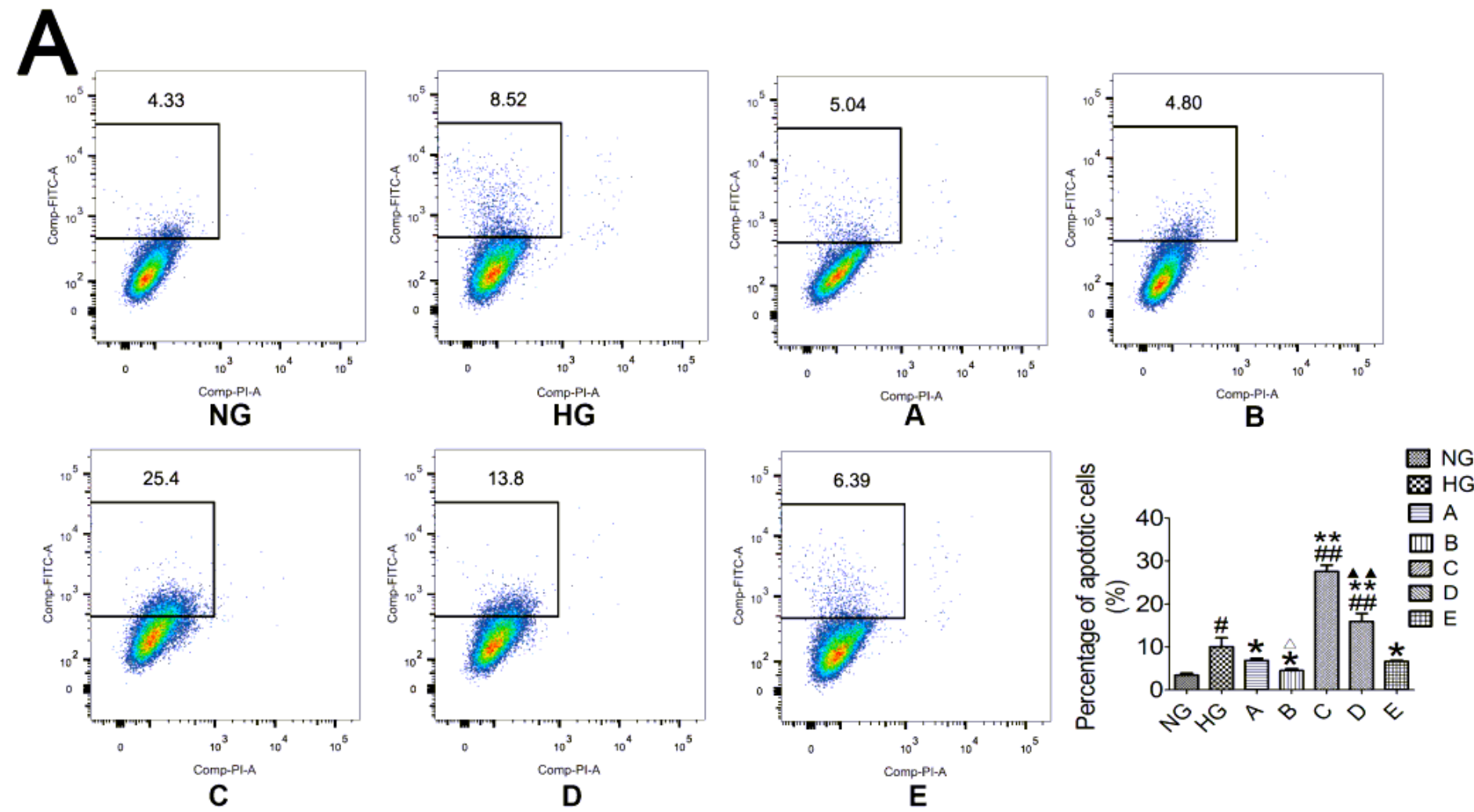

B

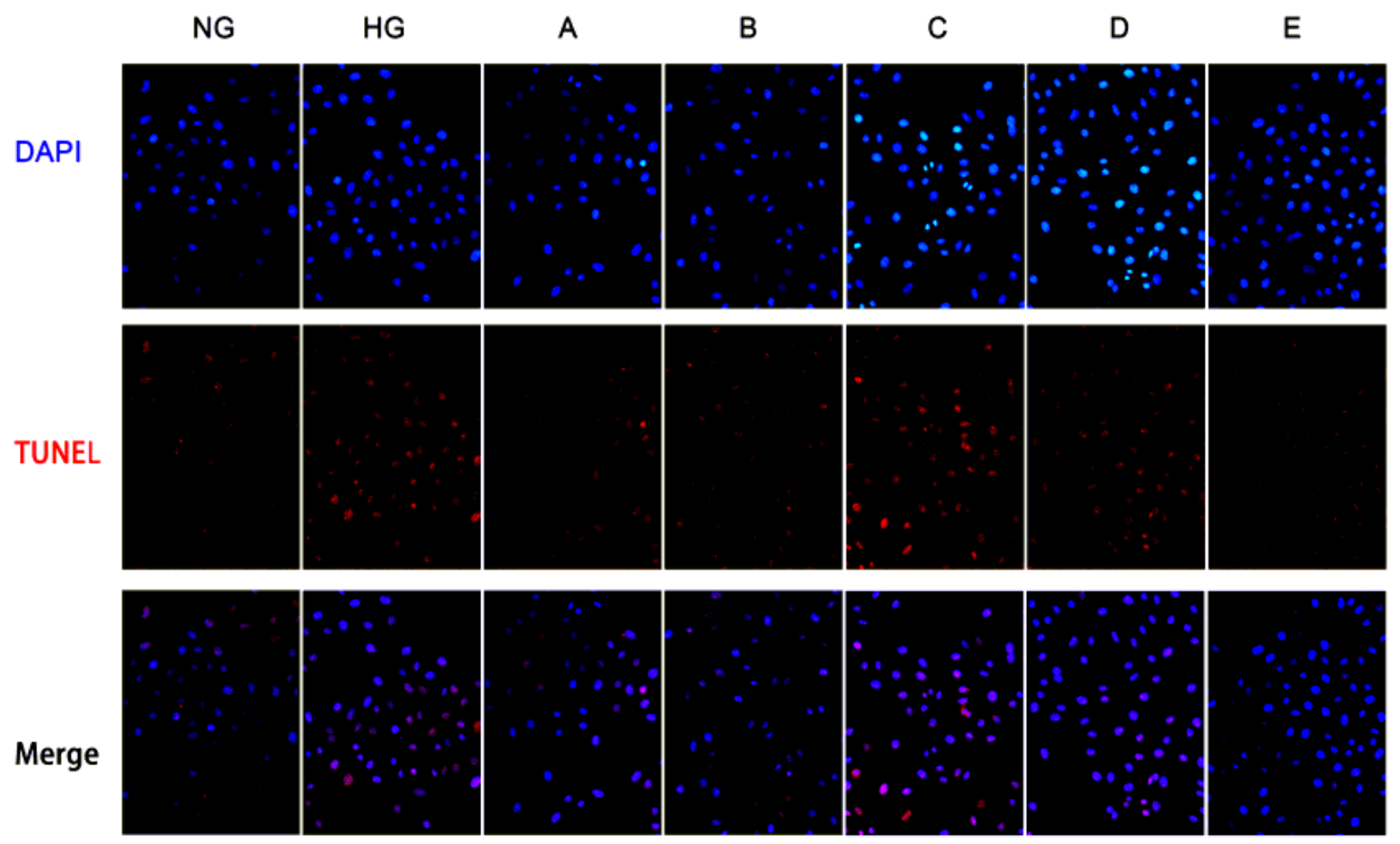

\section{Figure 5}

The effect of TRL on apoptosis in HMCs. (A) Representative flow cytometry graphs are shown. Early apoptotic cells are located in the grid, and the numbers above them represent the percentage of apoptotic cells. (B) DNA fragmentation in HMCs detected by TUNEL (200x). Cells that were positive for terminal deoxynucleotidyl transferase-mediated deoxyuridine triphosphate-biotin nick end-labeling (TUNEL) exhibited red staining in the nucleus. DAPI solution was used to stain nuclei (blue). NG: normal glucose, 
HG: high glucose, A: TRL, B: TRL+ $\beta$-catenin siRNA, C: $\beta$-catenin pcDNA, D: TRL+ $\beta$-catenin pcDNA, E: ICG001 ; \#\#P<0.01 versus NG; ${ }^{*} P<0.05$ versus $H G$, ${ }^{\star *} P<0.01$ versus $H G, \triangle P<0.05$ versus $A ; \Delta \Delta P<0.01$ versus C. 